\title{
Zur Lokalisation der Wortfindung bzw. der amnestischen Aphasie.
}

\author{
Von
}

A. Pick.

(Eingegangen am 20. April 1914.)

In einer ergebnisreichen, vorwiegend der amnestischen Aphasie gewidmeten Arbeit kommt Kehrer (Beitrag z. Aphasielehre 1913, S. 148) bezüglich dieser zu dem Schlusse, daß eine gesicherte Lokalisation für dieselbe nicht existiert. Da ich nun zu denjenigen gehöre, die doch eine solche im Schläfelappen postulieren, sehe ich mich veranlaßt, zu Kehrers Ansicht Stellung zu nehmen. Aber noch ein anderer Grund veranlaßt mich dazu; Kehrers These könnte, da sie sich als das Endergebnis einer Arbeit darstellt, die auch ein besonderes Kapitel: „Zur pathologischen Physiologie der amnestischen Aphasie" enthält, auch nach einer anderen Richtung hin zu Folgerungen Veranlassung geben, die nicht unwidersprochen bleiben können.

Trotz der besonders von v. Monakow ausführlich behandelten Differenzierung zwischen Lokalisation der Krankheit und der der Funktion könnte Kehrers These auch in dem Sinne aufgefaßt werden, als ob sie auch für die amnestische Funktion gelten würde. Nun bin ich geneigt, auf Grund der in meinem Buche: „Die agrammatischen Sprachstörungen“ gegebenen Darstellung des „Weges vom Denken zum Sprechen" den Sitz dieser Funktion in den Schläfelappen, etwa in die basalen Randpartien des akustischen Sprachfeldes zu verlegen, was nun, gleichfalls durch die These Kehrers als widerlegt angesehen werden könnte und deshalb zu einer Stellungsnahme zu dieser nötigt.

Zunächst möchte ich das von Kehrer selbst seiner Argumentation unmittelbar vorangesetzte Moment einer Besprechung unterziehen, um zu zeigen, daß die von ihm gezogene Konklusion doch auch anders ausfallen bzw. geradezu im Sinne einer gesicherten Lokalisation der mnestischen Funktion verwertet werden könnte. Kehrer erklärt die Tatsache des nicht isoliert über Jahre hinaus zu beobachtenden Vorkommens der a. A. ${ }^{1}$ ) daraus, da B sie eine der leichtesten Störungen darstellt und die in ihr betroffene Funktion eine der feinsten, daher vulnerabelsten Verbindungen zwischen Denken und Sprechen darstellt.

1) a. A. = amnestische Aphasie. 
Dieses Argument und die aus ihm gezogene Konklusion sind doch auch anderer Deutung zugänglich. Man kann zugeben, daß die a. A. eine der leichtesten Störungen, aber eben daraus folgern, daß die Läsionen dauerhafter Art, die zumeist das Material der Aphasielehre bilden, eben so intensiv wirken, daß die so zarte mnestische Funktion meist nicht allein geschädigt sein kann, ihre Störung also isoliert erst hervortritt, wenn sich die schweren, jene überdeckenden Erscheinungen zurückbilden; oder andernfalls, die die a. A. hervorrufende Störung ist eine derartig leichte, daß wohl auch ihre sonstige Wirkung eine leichte, damit aber auch die zeitliche Dauer der mnestischen Störung eine sehr begrenzte sein muß. Und das letztere läßt sich aus der Kasuistik direkt als zutreffend erweisen; die primär reinsten Fälle sind durch pachymeningitische (traumatische) Blutungen bedingt, die den Schläfelappen selbst betroffen haben oder (was man bisher bei der Kritik entsprechender Fälle nicht genügend gewürdigt) durch nachträgliche Senkung in die mittlere Schädelgrube die gleiche Wirkung dort ausüben.

Von diesen Tatsachen aus könnte man aber auf Grund der von mir sog. ,psychologischen Lokalisation" noch weiter argumentieren. Die Wortfindung ist eine der am weitesten vorwärts auf dem akustischen Anteil des Weges vom Denken zum Sprechen gegen den motorischen Anteil desselben vorgeschobenen Etappen und das stimmt damit überein, daß die ihr angenommenermaßen entsprechende Partie des Schläfelappens recht exzentrisch von der Wernickeschen Stelle, in der 2. bzw. 3. Temporalwindung, gelegen ist.

Ich möchte aber weiter betonen, daß das Argument Kehrers von der Dignität der mnestischen Funktion als einer besonders feinen, doch nicht so gefestigt dasteht, daß dieser Umstand nicht auch anders erklärt werden könnte. Das könnte zunächst damit zusammenhängen, daß sie in den obersten Rindenschichten des betreffenden Abschnittes lokalisiert sein möchte und dadurch selbst schwachen Einwirkungen leichter zugänglich wäre ${ }^{1}$ ).

Man muß aber für die Beurteilung des in Rede stehenden Momentes auch in Betracht ziehen, daß die Störung der Wortfindung ein sehr grobes und deshalb unseren, doch recht rohen klinischen Methoden sich alsbald präsentierendes Symptom darstellt, hinter das andere gewiß ebenso feine Störungen unbeachtet zurücktreten. Und damit stimmt auch überein, was wir bezüglich der Dignität der verschiedenen einander auf dem Wege vom Denken zum Sprechen folgenden Vor-

1) Gewiß sind wir, seit ich in meinem Amsterdamer Vortrage (1908) für eine schichtweise Lokalisation eingetreten bin, mit den bezüglichen tatsächlichen Feststellungen nicht weiter gekommen, aber für theoretisch verwertbar in dem obigen Sinne halte ich das darüber Bekannte denn hoch. 
gänge $\left.{ }^{1}\right)$ annehmen dürfen, daß sie einander in Rücksicht des eben besprochenen Gesichtspunktes vielleicht gleich sind.

Aber selbst für den Fall, daß Kehrers Annahme die richtige, läßt sich daraus doch folgern, daß die Leichtigkeit, mit der eben die mnestische Funktion beim Sprechen von überall her in Mitleidenschaft gezogen wird, nicht gegen die Lokalisation in einem bestimmten Territorium spricht; gerade wegen der Zartheit dieser Funktion, kann man sagen, wird sie von überallher besonders leicht in Mitleidenschaft gezogen; darin liegt gewiß keine Überspannung des Prinzips der Diaschisis ${ }^{2}$ ). Durch denselben Gedankengang wird es auch verständlich, wie gerade bei den sog. transcorticalen Formen Störungen der Wortfindung besonders hervortreten, weil bei diesen Formen die Diaschisis vor allem in der Richtung der zentrifugal ablaufenden Bahn, die in meinem Sinne dem Wege vom Denken zum Sprechen entspricht, wirksam sein wird.

Daß mit diesen Erwägungen doch auch die von Kehrer aufgestellten Formen der Wortamnesie, diejenige bedingt durch Störung der inneren Wortklangerweckung oder die infolge gestörter Utbertragung des Wortklangs auf das Motorium, glatt vereinbar sind, bedarf nicht erst des Nachweises.

Einzelne im Vorangehenden geäußerte Erwägungen können auch gegenüber der Stellung gelten, die neuestens v. Monakow in seiner Zusammenfassung der "Lokalisation im Großhirn“ 1914, S. 8433) einnimmt; doch muß unsere Stellungsnahme noch etwas breiter zur Darstellung gebracht werden, weil gewisse, wenn auch nur anmerkungsweise gemachte Äußerungen v. Mona kows in schroffstem Widerspruch zu dem Standpunkte stehen, den wir in der Frage der Lokalisation zu dem unseren gemacht.

Wenn v. Monakow (l. c. S. 845 Anm.) Ham mond ${ }^{4}$ ) darin Recht gibt, daß jeder Herd, zumal in der hinteren Sprachregion, mag er sitzen

1) Man beachte auch den Gegensatz zwischen dieser von mir vertretenen Ansicht und der älteren noch von Kehrer benützten, der zufolge die mnestische Funktion ,eine der Verbindungen zwischen Denken und Sprechen darstellt".

2) In diesem Zusammenhange möchte ich eine Deutung anführen, die B yrom Bramwell (Brain P. 83, S. 369) zur Erklärung einer als Standardfall zitierten, bei Zerstörung der Broca-Stelle vorhanden gewesenen amnestischen Aphasie gegeben: "The most probable explanation of the inability to name objects and persons is, it seems to me, to suppose that the action of that part of the auditory speech-centre in which names (highly specialised parts of speech) are stored and recalled (or, as Broadbent and Mills would say, of a special centre other than the auditory speech-centre, (the ,naming " centre, as they term it) was deranged by the lesion in the motor-vocal speech-centre."

3) So z. B. wenn er (1. c. S. 844) die isolierte amnestische Aphasie nur als Durchgangsstadium der kompletten sensorischen Aphasie gelten läßt.

$\left.{ }^{4}\right)$ Im Original findet sich übrigens die schroffe Formulierung des Satzes nicht. 
wie immer, den Mechanismus der Sprache derart desorganisieren kann, daß jede beliebige Form von sensorischer Aphasie auftreten kann, so ist, wie gesagt, damit ein Widerspruch gegenüber der hier vertretenen Ansicht markiert, der einer Klärung dringend bedarf; denn so genommen, könnte er im Sinne der Unmöglichkeit jeder Lokalisation sensorischaphasischer Störungen gedeutet werden; dann wird er aber ebenso durch die Erfolge der letzten Jahrzehnte, aber noch mehr durch deren theoretische Grundlagen widerlegt. Der zitierte Satz läßt zwei Deutungen zu: die Kombination von unmittelbaren Ausfallserscheinungen, Nachbarschaftssymptomen, Fernwirkungen und Diaschisis oder wie sonst man die andere Serie von Erscheinungen bezeichnen will, sind derartige, da $B$ sie trotz der Bemühungen seit Nothnagel und Wernicke jeder "festen Lokalisation" insbesondere des in Rede stehenden Symptoms widerstreben. Ich $\mathrm{mu}$ ß gestehen, dem vorliegenden Materiale dazu, namentlich „Herd“ im Sinne von Erweichung und stationär gewordener Blutung genommen, den gegensätzlichen Eindruck abgewonnen zu haben und auch durch v. Mona kows neueste Darstellung selbst nicht eines anderen belehrt worden zu sein. Gewiß wird v. Mona kow dagegen einwenden, daß er immer nur von klinischer Lokalisation spricht; aber ich möchte dem entgegenhalten, daß, wenn wie hier der Gesichtspunkt der Funktionslokalisation trotz vermeintlich noch nicht genügend bereinigter Symptomenlokalisation doch an der Hand der letzteren schon befriedigend in Betracht gezogen werden kann, dies nicht bloß ein berechtigter, sondern direkt ins Auge zu fassender Standpunkt ist. Und das führt zu der Widerlegung der zweiten, nicht unmöglichen Deutung, die dem zitierten $\mathrm{Ham}$ mondschen Ausspruche gegeben werden kőnnte. Unter dem Gesichtswinkel der Funktionslokalisation betrachtet und unter derVoraussetzung, daß uns die zuvor erwähnten erschwerenden diagnostischen Momente doch nicht mehr ganz undurchsichtig sind, würde jener Satz nämlich nichts anderes besagen als, daß eine Funktionslokalisation im Schläfelappen überhaupt aussichtslos ist, vielleicht sogar, weil sie in präziser Weise überhaupt nicht vorhanden ist. Dem möchte ich aber prinzipiell widersprechen und noch hinzusetzen, daß auch das Wenige der von mir sog. psychologischen Lokalisation auf dem Wege vom Denken zum Sprechen in befriedigendem und viel versprechendem Einklange mit der klinischen Lokalisation, gerade in jener kompliziertesten Region steht.

Wenn v. Monakow in der prinzipiellen Zusammenfassung des Materiales (l. c. S. 852) zu einer gegensätzlichen Ansicht bezüglich der Lokalisation der a. A. kommt, so schließen auch seine Argumente eine Deutung im Sinne der hier vertretenen Theorie, in der die Funktionslokalisation als die der optimalen aus der klinischen gefolgert wird, nicht aus. 
Gewiß ist es unbestreitbar, daß wie v. Mona kow bei der Erörterung der allgemeinen Prinzipien der Lokalisation (l. c. S. 2) darlegt, die Örtlichkeit, von welcher aus bestimmte Leistungen geschädigt werden, nur wenig Näheres aussagt in bezug auf die wirkliche zentrale Repräsentation derjenigen physiologischen Faktoren, welche durch die örtliche Läsion eine Beeinträchtigung erfahren. Aber, um bei dem von ihm gewählten Vergleiche mit dem Chronometer zu bleiben, der durch ein in das Räderwerk eingedrungenes Stäubchen in seinem Gange gestört ist, es gelingt dem Kenner, allmählich auf dem Wege kombinierter induktiver und deduktiver Methode (und als solche stellt sich die dar, die wir zuvor als die der optimalen Lokalisation bezeichnet haben) zu präzisieren, wo das Stäubchen sitzen muß, um zunächst etwa ein kleines Rädchen und von diesem aus das ganze Räderwerk zum Stillstand zu bringen; und ebenso muß es gelingen, das gegenüber anderen Schädigungen, z. B. durch Bruch einer Schraube und ähnliches zu differenzieren. Wenn dann v. Monakow betont, daß wir, um zu einer Funktionslokalisation zu gelangen, zunächst einer nach wissenschaftlichen Prinzipien und nicht nur nach einer populären Betrachtungsweise vorgenommenen Analyse der örtlichen Reiz- und Ausfallserscheinungen bedürfen, wenn er an einer anderen Stelle von der ,alltagspsychologischen Betrachtungsweise“ spricht, so fällt das im wesentlichen zusammen mit dem von mir in meinen ,Agrammatischen Sprachstörungen" gemachten Versuche, die neuen Fundamente der Aphasielehre in der modernen wissenschaftlichen Psychologie und Linguistik nachzuweisen.

Von den hier dargelegten Gesichtspunkten aus halte ich es doch für möglich, die Schlußsätze v. Monakows über die Lokalisation der a. A. wesentlich, sagen wir, optimistischer zu fassen.

Zunächst möchte ich die von ihm angeführten funktionell zu deutenden Fälle aus der Zahl der etwas Bestimmtes beweisenden ausschalten und ebenso die bei ausgedehnter Arteriosklerose ,ohne nennenswerte örtliche Läsion"; den ersteren kommt nur dann irgendwelche Beweiskraft zu, wenn der Gang der Störungen mit den aus groben Läsionen resultierenden Schlüssen in Einklang gebracht werden kann; im Gegensatze zu der Konklusion v. Monakows wird man doch auch nicht übersehen können, daß die von Hughlings Jacksons „Uncinate type“ und „dreamy states" abzuleitenden Schlüsse für die engere Lokalisation der Wortfindung im Schläfelappen sprechen.

Das von der Arteriosklerose hergenommene Argument gegen eine solche wird kaum widerspruchslos hinzunehmen sein. Finden wir doch bei ihr unzweifelhaft lokalisierbare Symptome, z. B. die Parästhesien im Ulnarisgebiet, ohne daß dafür eine besonders nennenswerte örtliche Läsion nachweisbar wäre. Die von v. Monakow angeführten 
Fälle von Schädeltrauma und speziell die Schädelbasisfrakturen sprechen meines Erachtens nichts gegen die hier vertretene doch vorwiegend zum Schädel basale Lokalisation der a. A. Gibt doch v. Monakow selbst zu, daß diese häufiger und intensiver als von anderen Stellen des Großhirns nach Läsion des Gyr. occipitotempor. zustande kommt.

Damit bietet er selbst einen sagen wir ersten Ansatz zu einer Betrachtung im Sinne unserer optimalen Methode und die von ihm in dem erwähnten Buche mitgeteilten neuen Fälle bieten dazu weitere prägnante Handhaben ${ }^{1}$.

Auf die Anschauungen v. Monakows bezüglich der bei der a. A. in Betracht kommenden weiteren Erregungskreise hier näher einzugehen, würde zu weit führen; es mag nur angedeutet werden, daß die entgegengesetzte Ansicht von den engeren Zentren gerade in Kehrers Thesen eine Stütze findet, insofern durch sie das von v. Monakow einheitlich gedeutete Symptom der Wortamnesie doch wieder in funktionelle Komponenten zerlegt wird; dadurch ist es aber ermöglicht, jeweils zur Erklärung eingeschränktere und ausgedehntere Erregungskreise heranzuziehen.

Es kann hier auch nur angedeutet werden, daß bei einer im Sinne der von mir vertretenen Funktionspsychologie gegenüber der bisher in der Aphasielehre herrschenden Lehre von den da oder dort „niedergelegten Erinnerungsbildern" geübten Betrachtung der mnestischen Funktion der scheinbar so schroffe Gegensatz der hier vertretenen Lokalisation zu derjenigen $\mathrm{v}$. Mo na kow $\mathrm{s}$ wesentlich gemildert erscheint. Eine Unterstützung findet die Nutzanwendung dieses Gesichtspunktes andererseits dadurch, daß die Wortfindung an der Hand der neueren Psychologie analysiert, sich doch wesentlich komplizierter darstellt als es die bisher übliche alltagspsychologische Betrachtungsweise (Assonanz von Objekt- und Wortbegriff) glauben machen wollte.

Zum Schlusse möchte ich noch darauf hinweisen, daß ich mit der von mir als der optimistischen bezeichneten Anschauung von der Möglichkeit einer schärferen Lokalisation der a. A. doch nicht so ganz allein stehe, wie es insbesondere nach der Lektüre der zitierten Stelle des Buches v. Monakows scheinen könnte, in dem die Gegner jener Ansicht mehr zum Worte kommen; dagegen lautet eine andere Äußerung²) v. Monakows (1. c. S. 613) trotz aller angeschlossenen Vorbehalte doch ganz im Sinne der hier vertretenen Ansicht. Daß es sich dabei aber nicht um eine Doktorfrage sondern um eine solche von eminent

1) Dazu rechne ich auch die zwei von v. Stauffenberg aus v. Monakows Institut veröffentlichten Fälle von Seelenblindheit, die beide infolge von Schläfelappenherden amnestische Aphasie, der eine fast rein, aufwiesen.

2) „Bei fortschreitenden Herden besonders in bestimmten Windungsgebieten (z. B. im Mark $\mathrm{T}_{2}$ und $\mathrm{T}_{3}$ ) tritt die Wortamnesie in überaus typischer Weise auf." 
290 A. Pick: Zur Lokalisation der Wortfindung bzw. der amnestischen Aphasie. praktischer Bedeutung handelt - ich erinnere nur an die otitischen Hirnabscesse - beweist gerade die ihr von v. Monakow gegebene Formulierung.

Nachträgliche Bemerkung nach Absendung des Manuskripts: In Hinsicht des zuletzt Gesagten entnehme ich dem mir jetzt nachträglich bekannt gewordenen Berichte über die 100. Sitzung des ostdeutschen Vereins für Psychiatrie (Zeitsch. f. Psych. 71), daß Stertz, Bonhoeffer und $O$. Foerster im wesentlichen mit der von mir vertretenen Ansicht übereinstimmen. 